\title{
界面调控二维六方氮化硼单晶外延生长取得新进展
}

\author{
吴克辉 \\ 中国科学院物理研究所, 表面物理国家重点实验室, 北京 100190 \\ E-mail: khwu@iphy.ac.cn
}

\section{New progress of the epitaxy of two dimensional single-crystal hexagonal boron nitride by interface regulation}

\author{
Kehui Wu \\ State Key Laboratory of Surface Physics, Institute of Physics, Chinese Academy of Sciences, Beijing 100190, China \\ E-mail: khwu@iphy.ac.cn
}

doi: 10.1360/TB-2019-0194

近年来, 随着芯片内元器件尺寸的不断减小, 短沟道 效应、热效应等日趋明显, 开发全新的二维量子材料体系 以实现变革性的器件应用已成为当前的研究热点. 高端器 件的规模化应用必须基于大面积、高品质的单晶材料, 因 此二维单晶材料的制备研究具有重要的科学意义和技术 价值. 2019年5月22日, Nature在线发表了题为“Epitaxial growth of a 100-square-centimetre single-crystal hexagonal boron nitride monolayer on copper”的文章, 报道了北京大 学物理学院刘开辉研究组及其合作者 ${ }^{[1]}$, 利用表面中心反 演对称性破缺的单晶铜衬底实现了分米级单晶六方氮化 嗍的外延制备. 该项研究成果受到学术界和工业界的广泛 关注.

硅基半导体器件的出现推动了现代信息化社会的发 展. 近年来, 随着晶圆制造及微纳加工工艺的不断完善, 芯片中元器件的集成度越来越高. 在芯片性能飞速提升的 同时, 线宽变小所带来的短沟道效应、热效应也严重阻碍 了芯片性能进一步发展. 然而, 从另一个角度来看, 小尺 度下显著的量子效应, 也为全新量子器件的研制打开了一 扇新的大门. 作为量子材料中的典型代表, 二维材料具有 原子级的厚度、完美的表界面以及优异的理化性质, 并且 体系丰富, 含导体 (石墨烯、嗍烯) ${ }^{[2,3]}$ 、半导体(过渡金属硫

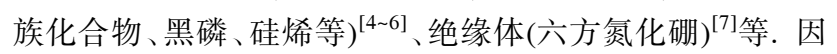
此, 全二维材料量子器件有望替代传统硅基器件, 在微电 子领域实现革命性突破.

规模化的器件应用需要大尺寸、高质量的单晶作为基 础. 二维单晶的制备方法与生长机理一直以来都是相关领 域研究的热点. 现有研究表明, 采用化学气相沉积法在单 晶衬底上外延得到的单晶二维材料可以较好地兼顾品质
与尺寸. 2017年, 王恩哥、俞大鹏、刘开辉课题组 ${ }^{[8]}$ 利用单 晶 $\mathrm{Cu}(111)$ 铜箔退火技术和石墨烯超快外延生长技术成功 在世界上首次实现了米级单晶石墨烯的制备, 将二维导体 石墨烯单晶推向了规模化应用的尺度. 遗憾的是, 传统的 外延生长方法并不适用于六方氮化嗍单晶薄膜的生长. 主 要原因是六方氮化硼是二元化合物, 与石墨烯不同, 其六 角形晶格不具有中心反演对称性, 在大多数表面具有中心 反演对称性的常规衬底上外延生长时会出现两个对称(间 隔 $180^{\circ}$ 转角)的等价优势取向(图1(a)，(b)). 这两种取向的 晶畴在拼接的过程中会形成孪晶晶界, 进而形成多晶薄 膜 ${ }^{[9]}$.

解决这一难题的关键在于衬底对称性的调控. 北京 大学刘开辉团队及其合作者 ${ }^{[1]}$ 设计出了一种具有特殊台阶 方向的晶面. 通过台阶与六方氮化嗍不同晶格方向匹配的 能量差异, 打破褯表面的中心反演对称性, 进而打破两 个优势取向的能量简并, 从而实现了六方氮化嗍晶畴的单 一取向生长, 并且无缝拼接为整片单晶(图1(c)).

具体来讲, 他们首先利用接近熔点的温度退火使得 铜䈃表面预熔, 以提高 $\mathrm{Cu}(110)$ 晶面成核的概率; 然后再利 用温度梯度驱动使得 $\mathrm{Cu}(110)$ 晶面长大, 最终得到 $(10 \times 10)$ $\mathrm{cm}^{2}$ 的单晶铜管. 轻微氧化后可发现 $\mathrm{Cu}(110)$ 的区域颜色较 深, 作为参照 $\mathrm{Cu}(111)$ 的区域颜色比较亮(图2(a)), 这是由 不同晶面指数铜䇴的氧化势垒不同所导致的. 在得到的 $\mathrm{Cu}(110)$ 单晶铜箔上生长六方氮化硼, 可以发现所有的六 方氮化嗍晶畴都是取向一致的(图2(b)). 低能电子衍射的 结果可以确认, 在单晶 $\mathrm{Cu}(110)$ 上六方氮化硼晶格取向也 是一致的(图2(c), (d)). 为了深人了解 $\mathrm{Cu}(110)$ 晶面上台阶 调控六方氮化嗍生长的机理, 他们还利用扫描探针显微技 
术发现六方氮化嗍晶畴的长边(对应晶格的zigzag方向)与 台阶边缘平行(图2(e), (f)). 结合低能电子衍射结果(图2(c), (d)) 分析可知, 六方氮化嗍晶格的zigzag边与 $\mathrm{Cu}(110)$ 晶面 上<211>台阶方向平行(图2(g)). 通过第一性原理的计算结
果发现, 六方氮化嗍晶畴中B-zigzag $\left(\gamma= \pm 60^{\circ}\right)$ 和 N-zigzag $\left(\gamma=0^{\circ}\right)$ 与 $\mathrm{Cu}<211>$ 台阶耦合的形成能存在较大的差异, 打 破了沿台阶成核过程中正向与反向 (180转角)晶畴的能量 简并, 进而实现了晶畴同取向生长并无缝拼接为大面积、

(a)

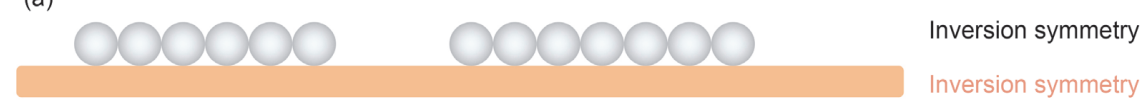

(b)

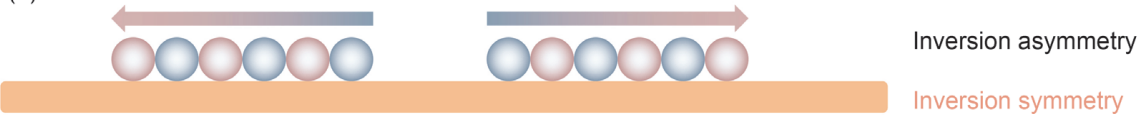

(c)

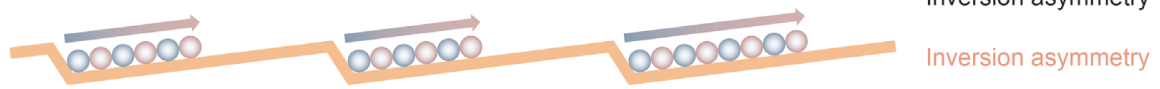

图 1 (网络版彩色)利用对称性破缺祄底外延单晶的设计思路. (a) 在中心反演对称性衬底上外延中心反演对称性材料, 所有晶畴取向一致; (b) 在中心反演对称性衬底上外延非中心反演对称性材料, 晶畴存在两个间隔 $180^{\circ}$ 的取向; (c) 在非中心反演对称性衬底上外延非中心反演对称性 材料, 所有晶畴取向一致

Figure 1 (Color online) Design of the epitaxy on the symmetry broken substrate. (a) Unidirectionally aligned epitaxy of the material with inversion symmetry on the substrate with inversion symmetry; (b) epitaxy of the material without inversion symmetry on the substrate of inversion symmetry, which brings two domain orientations with rotation angle of $180^{\circ}$; (c) unidirectionally aligned epitaxy of the material without inversion symmetry on the substrate of inversion asymmetry
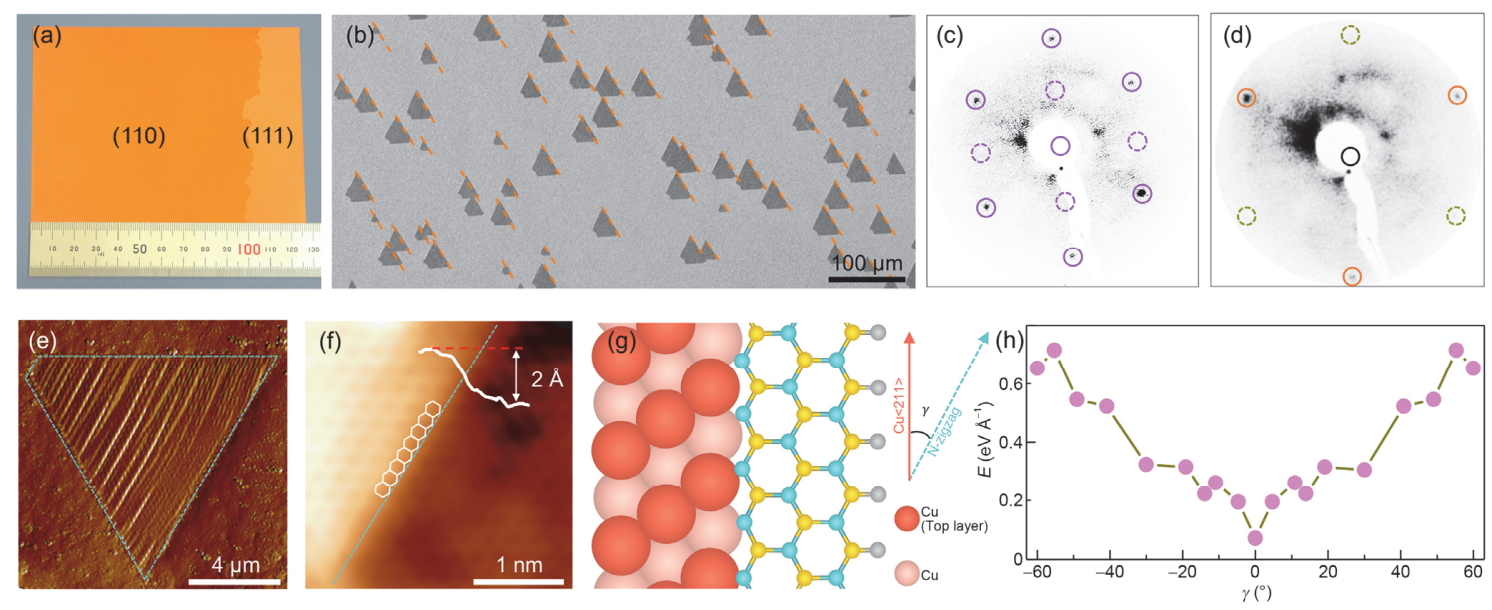

图 2 (网络版彩色)单晶六方氮化嗍的生长、表征及生长动力学研究. (a) 单晶铜簿的光学照片; (b) 取向一致的六方氮化硼晶畴的扫描电子显微 镜照片; (c) 单晶 $\mathrm{Cu}(110)$ 的低能电子衍射图案; (d) 单晶六方氮化嗍的低能电子衍射图案; (e) $\mathrm{Cu}(110)$ 上六方氮化嗍晶畴的原子力显微镜扫描; (f) $\mathrm{Cu}(110)$ 上六方氮化嗍晶畴的扫描隧道显微镜照片; $(\mathrm{g})$ 氮化硼晶格和 $\mathrm{Cu}(110)$ 晶面上 $<211>$ 方向台阶构型的示意图; (h) 不同氮截止锯齿边与 $\mathrm{Cu}<211>$ 夹角情况下台阶调控六方氮化硼生长的形成能

Figure 2 (Color online) Epitaxy, characterization and growth kinetics of single-crystal hexagonal boron nitride. (a) Image of single-crystal copper foil; (b) scanning electron microscope image of as-grown unidirectionally aligned hexagonal boron nitride domains; (c) low energy electron diffraction pattern of single-crystal $\mathrm{Cu}(110)$ foil; (d) low energy electron diffraction pattern of single-crystal hexagonal boron nitride; (e) atomic force microscope scan of hexagonal boron nitride on $\mathrm{Cu}(110)$; (f) scanning tunneling microscope image of hexagonal boron nitride on $\mathrm{Cu}(110)$; ( $\mathrm{g}$ ) schematic diagrams of the configuration of the hBN lattice and the atomic step along with $<211>$ direction on $\mathrm{Cu}(110)$; (h) formation energies of edge-guided growth of hexagonal boron nitride with respect to different rotation angle between $\mathrm{N}$-zigzag and $\mathrm{Cu}<211>$ direction 
高质量的六方氮化嗍单晶薄膜.

事实上, 相当多数的二维材料晶格都是非中心反演 对称性的, 它们的单晶尺寸是限制二维材料器件从实验室 走向大规模工业化应用的瓶颈难题. 刘开辉研究组与合作
者提出了利用对称性破缺祄底实现二维单晶生长的新思 路，具有广泛的借鉴意义，可推广至其他二维材料的大尺 寸单晶制备. 该工作为后续二维量子器件的研究奠定了坚 实的材料基础, 有望推进和带动相关产业的发展.

\section{参考文献}

1 Wang L, Xu X, Zhang L, et al. Epitaxial growth of a 100-square-centimetre single-crystal hexagonal boron nitride monolayer on copper. Nature, 2019, 570: 91-95

2 Geim A K, Novoselov K S. The rise of graphene. Nat Mater, 2007, 6: 183-191

3 Feng B, Zhang J, Zhong Q, et al. Experimental realization of two-dimensional boron sheets. Nat Chem, 2016, 8: 563-568

4 Wang Q H, Kalantar-Zadeh K, Kis A, et al. Electronics and optoelectronics of two-dimensional transition metal dichalcogenides. Nat Nanotechnol, 2012, 7: 699-712

5 Takao Y, Asahina H, Morita A. Electronic structure of black phosphorus: Tight binding approach. J Phys Soc Jpn, 1981, 50: 3362-3369

6 Liu C C, Jiang H, Yao Y. Low-energy effective Hamiltonian involving spin-orbit coupling in silicene and two-dimensional germanium and tin. Phys Rev B, 2011, 84: 195430

7 Kubota Y, Watanabe K, Tsuda O, et al. Deep ultraviolet light-emitting hexagonal boron nitride synthesized at atmospheric pressure. Science, 2007, 317: 932-934

8 Xu X, Zhang Z, Dong J, et al. Ultrafast epitaxial growth of metre-sized single-crystal graphene on industrial Cu foil. Sci Bull, 2017, 62: $1074-1080$

9 Song X, Gao J, Nie Y, et al. Chemical vapor deposition growth of large-scale hexagonal boron nitride with controllable orientation. Nano Res, 2015, 8: 3164-3176 